\title{
REVIEWS
}

\section{Novel RAAS agonists and antagonists: clinical applications and controversies}

\author{
Cesar A. Romero, Marcelo Orias and Matthew R. Weir
}

\begin{abstract}
The renin-angiotensin-aldosterone system (RAAS) regulates blood pressure homeostasis and vascular injury and repair responses. The RAAS was originally thought to be an endocrine system critically important in regulating blood pressure homeostasis. Yet, important local forms of the RAAS have been described in many tissues, which are mostly independent of the systemic RAAS. These systems have been associated with diverse physiological functions, but also with inflammation, fibrosis and target-organ damage. Pharmacological modulation of the RAAS has brought about important advances in preventing morbidity and mortality associated with cardiovascular disease. Yet, traditional RAAS blockers such as angiotensin converting enzyme (ACE) inhibitors and angiotensin receptor blockers (ARBs) only reduce the risk of disease progression in patients with established cardiovascular or renal disease by $20 \%$ compared with other therapies. As more components of the RAAS are described, other potential therapeutic targets emerge, which could provide improved cardiovascular and renal protection beyond that provided by an ACE inhibitor or ARB. This Review summarizes the present and future pharmacological manipulation of this important system.
\end{abstract}

Romero, C. A. et al. Nat. Rev. Endocrinol. 11, 242-252 (2015); published online 10 February 2015; doi:10.1038/nrendo.2015.6

\section{Introduction}

More than seven decades ago, angiotensin, a polypeptide that is the product of the enzymatic activity of renin, was found in venous samples of ischaemic dog kidneys. ${ }^{1}$ In the 1950s and 1960s, two forms of angiotensin were reported: angiotensin-1 (Ang I), which is 10 amino acids long, and angiotensin-2 (Ang II), with 8 amino acids. ${ }^{2}$ The latter form results from the metabolism of Ang I by a dipeptidyl carboxypeptidase named angiotensinconverting enzyme (ACE). The substrate of renin was found to be angiotensinogen, a serum globulin produced by the liver. ${ }^{3}$ Also at this time, the role of Ang II in the regulation of aldosterone production by the adrenal cortex emerged. ${ }^{4}$

In the 1970s, the main catalytic cascade of the reninangiotensin-aldosterone system (RAAS) was described (Figure 1). ${ }^{5}$ Plasma angiotensinogen is cleaved by renal renin, producing Ang I, which is then converted to Ang II by endothelial ACE, a process that occurs most extensively in lung tissue. Ang II was considered the most important RAAS mediator, with increased levels of Ang II being associated with vasoconstriction and increased blood pressure. Ang II binds to the type-1 Ang II receptor (AT1) in a variety of tissues, including vascular smooth muscle and the adrenal grand, to mediate many mechanisms that lead to raised blood pressure. The stimulation of aldosterone production via the AT1 receptor in the adrenal gland facilitates sodium retention by the kidney when aldosterone binds to the mineralocorticoid receptor.

Competing interests

The authors declare no competing interests.
On the basis of the first experimental studies of Ang II, in which supraphysiological doses of this peptide were tested in dogs, the RAAS was related to the pathophysiology of hypertension mainly through the effects of this system on vascular constriction. ${ }^{1}$ However, more recent studies have helped clarify that blood pressure control by Ang II at physiological concentrations is more related to sodium and water handling than to arterial constriction. ${ }^{6}$ Ang II shifts the natriuresis-blood pressure curve, with increased levels of Ang II requiring increased blood pressure to eliminate the same quantity of sodium. Part of this effect occurs via direct mediation by Ang II, which activates sodium transporters in the proximal tubules of the kidney. ${ }^{7-9}$ Furthermore, Ang II, through binding to the AT1 receptors, is the most potent regulator of aldosterone secretion in the adrenal cortex. ${ }^{10,11}$ Aldosterone binding to the mineralocorticoid receptor induces nongenomic (rapid) and genomic effects in many tissues, but mainly in the kidney, where it increases the epithelial expression of epithelial sodium channel (ENaC, also known as amiloride-sensitive sodium channel) at the level of the distal tubules, which favours the retention of sodium and water. ${ }^{12}$

Blockade of the RAAS was a major breakthrough in the treatment of cardiovascular disease, lowering mortality and improving quality of life in patients with hypertension, chronic kidney disease (CKD), myocardial infarction and heart failure. ${ }^{13-17}$ The classic RAAS antagonists target the angiotensinogen-angiotensinAT1-aldosterone axis. However, as knowledge about the RAAS expands, the number of potential therapeutic targets in this system is increasing. In this Review we 


\section{Key points}

- Renin-angiotensin-aldosterone system (RAAS) blockade with an angiotensin converting enzyme inhibitor or an angiotensin receptor blocker provides a $20 \%$ relative risk reduction for the progression of established cardiorenal disease compared with other non-RAAS blocking therapies

- The RAAS is an endocrine, paracrine and autocrine system that regulates blood pressure homeostasis through effects on a variety of target organs, as well as having a role in the responses to vascular injury and repair

- The RAAS is a complex system with a variety of sites suitable for pharmacological intervention

- Novel molecules that alter the production of various RAAS peptides or that alter receptor density, function or responsiveness to these peptides could have an important influence on haemodynamics and vascular structure and function

discuss novel agonists and antagonists of the RAAS that might improve the care of patients with cardiovascular and renal disease.

\section{Components and function of the RAAS}

The components of the RAAS are schematically represented in Figure 1. Renin is the rate-limiting step in Ang II production and is released by the juxtaglomerular apparatus in the kidney in response to decreased renal perfusion pressure, low tubular salt load or sympathetic activation. Renin is synthesized from prorenin, a pre-proenzyme that is constitutively released, with plasma levels 10-fold higher than those of active renin. ${ }^{18}$ Prorenin can be proteolytically activated in the kidney by neuroendocrine convertase 1 (also known as prohormone convertase 1 and proprotein convertase 1) or cathepsin B and non-proteolytically (reversibly) in many tissues by the renin receptor (also known as renin/prorenin receptor). These two types of activation require the propeptide to expose its active site, a cleft between two similar domains. ${ }^{19}$ The renin receptor also binds to mature renin, increasing its catalytic activity by $4-5$ fold. ${ }^{19}$ The renin receptor can initiate Ang II-independent signalling pathways, such as the MAPK/ERK pathway and pathways involving the zinc finger protein PLZF. ${ }^{20}$ The effects of these signalling pathways are not clear and the renin receptor has not been shown to have a role in cardiovascular disease when the effects of Ang II are blocked. ${ }^{21}$

The secretion of renin is controlled by short-term and long-term negative feedback loops. Short-term regulation is mediated directly by Ang II through binding to AT1, which induces inhibition of renin gene expression. ${ }^{22}$ The long-term negative feedback loop is an indirect consequence of the effects of increased Ang II levels: increased blood pressure, sympathetic inhibition of baroreceptor mechanisms and increased intratubular sodium levels.

The classic effects of Ang II are mediated by the AT1 receptor, which is present in practically all tissues (Box 1). Blood pressure effects are modulated by vascular AT1 receptors, whose activity produces vasoconstriction (rapid pressure effects) and by renal AT1 receptors that mediate sodium and water reabsorption. Many fibrotic and inflammatory effects of Ang II are also a consequence of AT1 receptor activation. ${ }^{23}$ However, Ang II can also activate the type-2 Ang II receptor (AT2), which is more sparsely expressed in body tissues than the AT1 receptor, but which is upregulated in many cases of injury (for example, to the brain, heart and kidney). AT2 is part of the protective arm of the RAAS, and, when activated, has antifibrotic and anti-inflammatory effects (Box 1). Activation of the AT2 receptor does not seem to have blood pressure effects. ${ }^{24}$

Other non-classic RAAS components have been described in the past few years. A novel enzyme similar to ACE was identified and called angiotensin-converting enzyme 2 (encoded by ACE2 and hereby referred to as ACE2). ${ }^{25}$ This carboxypeptidase has great Ang II affinity and can transform this peptide to angiotensin 1-7 (hereby referred to as Ang 1-7). ACE2 can also convert Ang I to angiotensin 1-9 (Ang 1-9), which is then converted to Ang 1-7 by ACE. Similarly, plasmatic endopeptidases can convert Ang I to Ang 1-7. Ang 1-7 binds to a G-protein-coupled receptor called proto-oncogene Mas (encoded by MAS1 and hereby referred to as MAS1). ${ }^{26}$ This ACE2-Ang 1-7-MAS1 axis has been reported to function as a counterbalance to the classic ACE-Ang IIAT1 axis, with opposite effects than those mediated by the classic axis (Box 1). ${ }^{27}$

Another non-classic axis in the RAAS is the pathway mediated by angiotensin-4 (Ang IV) and its receptor, leucyl-cystinyl aminopeptidase (also known as insulin-regulated membrane aminopeptidase, insulinresponsive aminopeptidase or IRAP). ${ }^{28,29}$ This receptor, which was originally reported to colocalize with the glucose transporter GLUT-4, has a role in the degradation of vasopressin and oxytocin. Ang IV inhibits the enzymatic activity of IRAP and knockout models and studies of non-peptide inhibitors suggest a role of IRAP in cardiovascular disease, mainly in target-organ damage. ${ }^{30-32}$ The effects of IRAP inhibition are listed in Box 1 .

Beyond the original description of the endocrine roles of the RAAS, experimental studies have demonstrated that the system also has paracrine, autocrine and intracrine functions. Different tissues (reproductive and digestive tissues, heart and brain) and cells (adipocytes and white blood cells) express RAAS components, which not only perform the classic RAAS function (fluid volume homeostasis), but are also involved in other physiological functions, such as ovulation, ${ }^{33}$ secretion of insulin by the pancreas, ${ }^{34}$ cognitive function, ${ }^{35}$ inflammation $^{36}$ and cancer. ${ }^{37,38}$ In addition, activation of local RAASs is strongly related to target-organ damage, mostly in the kidney and heart. ${ }^{39}$

\section{Classic RAAS antagonists}

Four clinically established groups of drugs inhibit the RAAS: ACE inhibitors, angiotensin receptor blockers (ARBs, which block the AT1 receptor), renin inhibitors and mineralocorticoid receptor blockers (Box 2). Despite the important improvements achieved with these agents in slowing the progression of established cardiorenal disease, the ACE inhibitors and the ARBs only provide a $20 \%$ reduction in the relative risk of 


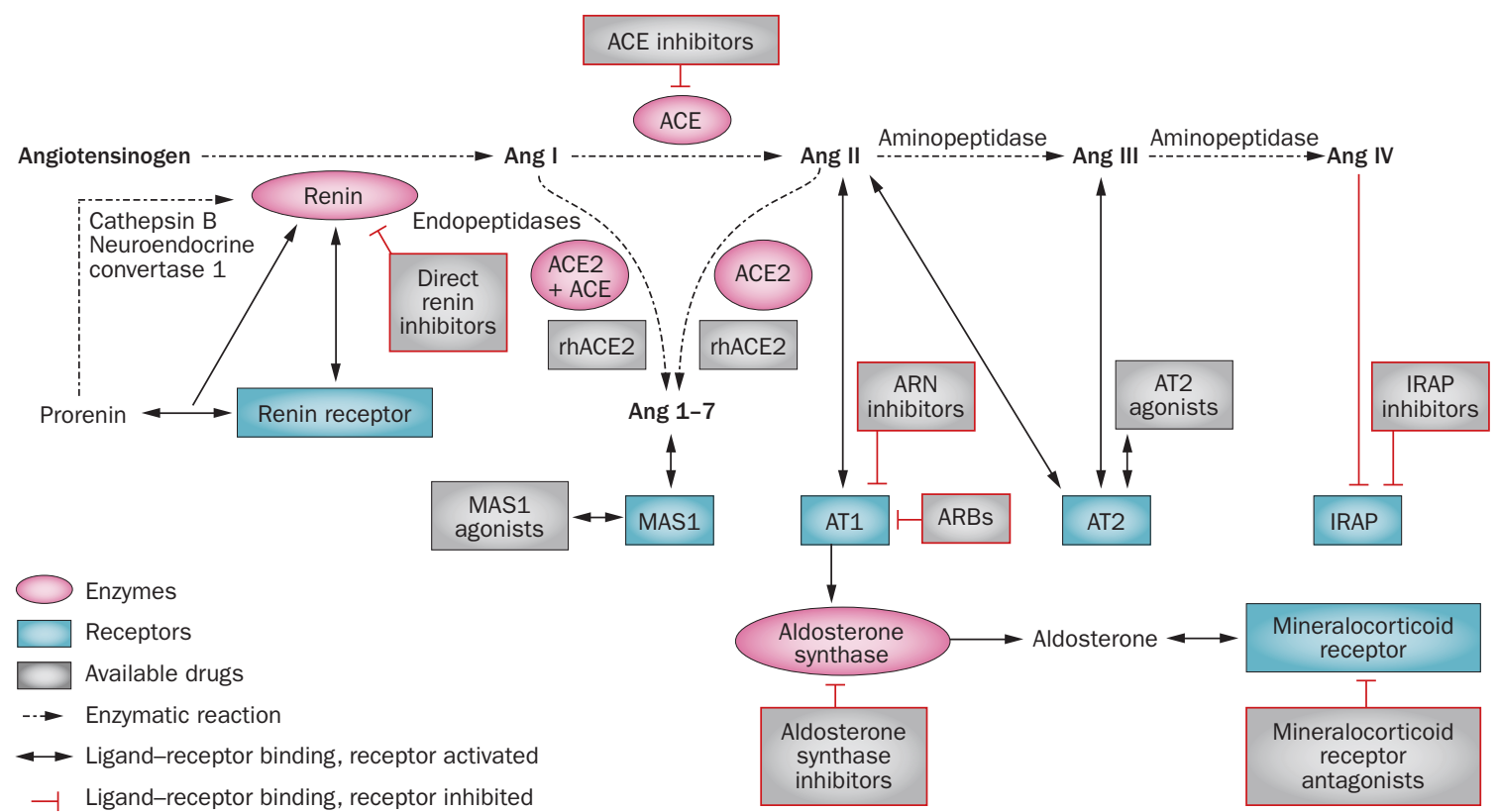

Figure 1 Components of the renin-angiotensin-aldosterone system and main classes of pharmacological activators and inhibitors of the system. Prorenin can be activated proteolytically in the kidneys (by neuroendocrine convertase 1 or cathepsin B) or nonproteolitically by the renin receptor in many tissues. Circulating renin can also bind to the renin receptor, which increases its enzymatic activity. Renin converts angiotensinogen to Ang I, which can then enter three main pathways. These three axes, ACE-Ang II-AT1-aldosterone, ACE2-Ang 1-7-MAS1 and Ang IV-IRAP, are highlighted. Activation of the AT1 receptor in the adrenal gland results in production of aldosterone, which can then bind to the mineralocorticoid receptor. Abbreviations: ACE, angiotensin-converting enzyme; ACE2, angiotensin-converting enzyme 2; Ang, angiotensin; ARB, angiotensin receptor blocker; ARN, angiotensin receptor-neprilysin; AT1, type-1 Ang II receptor; AT2, type-2 Ang II receptor; IRAP, leucyl-cystinyl aminopeptidase (also known as insulin-regulated membrane aminopeptidase or insulin-responsive aminopeptidase); MAS1, proto-oncogene Mas; rh, recombinant human.

progression of cardiovascular disease when compared with non-RAAS blocking therapy. ${ }^{14,16,17,40}$ Moreover, no data have demonstrated beneficial effects of ACE inhibitors or ARBs in the primary prevention of either cardiac or kidney disease. Therefore, much consideration has been given to how to improve upon RAAS inhibition with the classic, established drugs, by investigating new agonists and antagonists of the complex RAAS cascade. Many theories about why traditional RAAS inhibition has not provided more clinical benefits have been proposed, but none of these theories has been validated clinically.

\section{Inhibitors of the ACE-Ang II-AT1-aldosterone axis ACE inhibitors}

In the early 1980s, early clinical studies of the first oral ACE inhibitor, captopril, demonstrated this agent has antihypertensive properties and improves the clinical status of patients with heart failure. ${ }^{41} \mathrm{ACE}$ inhibitors do not affect the levels of ACE2. Even though the main mechanism of action of ACE inhibitors is to limit the formation of Ang II, other activities, such as reducing the catabolism of other vasodilator oligopeptides (such as bradykinin) by ACE, might have a role in the pharmacological effects of ACE inhibitors. Another tetrapeptide, $\mathrm{N}$-acetyl-seryl-aspartyl-lysil-proline (AcSDKP), whose levels are elevated in patients receiving ACE inhibitors, could also be responsible for many of the antifibrotic and immune-beneficial effects of these agents. ${ }^{42}$ Furthermore, ACE inhibitors increase the levels of Ang I, which shifts RAAS activity to the axis producing Ang 1-7.43,44 Some of the favourable pharmacological effects of ACE inhibitors might be mediated though increased activity of the ACE2-Ang 1-7-MAS1 axis. ${ }^{19,20}$

Currently, ten ACE inhibitors are available in the USA and several more are available globally. The clinical effects of the various ACE inhibitors are not markedly different, except for trandolapril and quinapril having a longer half-life and broader tissue distribution than the other agents owing to their liposolubility. Many years of use have confirmed very good safety and tolerability profiles for these drugs, with cough (6-20\% of patients) and angioedema ( $1 \%$ of patients) being the most common adverse effects. This drug class is the first-line therapy for patients with hypertension, CKD and heart failure, ${ }^{45-47}$ with ACE inhibitors successfully controlling blood pressure and reducing left-ventricular hypertrophy ${ }^{48}$ and all-cause mortality. ${ }^{49}$

\section{ARBs}

The first oral ARB, losartan, was made available in the 1990s, and currently nine ARBs exist for clinical use (Box 2). Despite different pharmacokinetic characteristics, ${ }^{50}$ most $\mathrm{ARBs}$ are used as once-daily drugs and have fewer adverse effects than ACE inhibitors. ${ }^{51}$ In general, the indications for using ARBs are the same as for using ACE inhibitors; ARBs are efficacious in hypertension ${ }^{52-54}$ and heart failure,,$^{55,56}$ and confer renal 
protection. ${ }^{57}$ As with ACE inhibitors, use of ARBs increases the levels of Ang II as a substrate of ACE2 and could have clinical benefits resulting from stimulation of the ACE2-Ang 1-7-MAS1 axis. ${ }^{19,20}$

In theory, combining $\mathrm{ACE}$ inhibitors with $\mathrm{ARBs}$ or with a renin inhibitor could have pharmacological benefits. However, no evidence of clinical benefits beyond those achieved with single-agent approaches was seen when combination therapy was used in patients with hypertension, coronary artery disease or CKD, and combination therapy could potentially be harmful. ${ }^{58,59}$

\section{Mineralocorticoid receptor antagonists}

The mineralocorticoid receptor antagonist spironolactone was first used in the 1960s as a potassium-sparing diuretic. Since then, the systemic effects of aldosterone in hypertension, heart failure and target-organ damage became better known. Randomized clinical trials confirmed the effectiveness of spironolactone to treat hypertension and showed that the drug has antifibrotic and anti-inflammatory properties. ${ }^{48}$ Spironolactone binds to other receptors in addition to the mineralocorticoid receptor, such as androgen and progesterone receptors, which can have adverse effects such as gynaecomastia and decreased libido. In 2002, a second mineralocorticoid receptor antagonist, eplerenone, was introduced. Eplerenone has less affinity for sex hormone receptors than spironolactone; the drug has a nearly similar antihypertensive effectiveness to that of spironolactone, yet is less potent and has fewer adverse effects. ${ }^{60,61} \mathrm{~A}$ third antagonist, canrenone, is available in some countries but scant clinical data on this drug exist. ${ }^{62}$ In addition to their use in primary hyperaldosteronism, mineralocorticoid receptor blockers are commonly used to treat ascites, ${ }^{63}$ heart failure ${ }^{64}$ and resistant hypertension. ${ }^{65,66}$

\section{Direct renin inhibitors}

In 2007, and after many attempts, the first oral direct renin inhibitor (DRI), aliskiren, became available, replacing first-generation inhibitors of low bioavailability. Aliskiren is an effective antihypertensive agent and dual therapy with aliskiren and an ARB was tested in the ALTITUDE trial. ${ }^{58}$ However, in this trial, the combination of aliskiren and losartan did not show any benefits on cardiovascular or renal outcomes versus losartan alone, and dual therapy was associated with an increased rate of adverse events. ${ }^{58}$

\section{Novel RAAS agonists and antagonists}

Improving the benefits of RAAS blockade beyond the effects of ACE inhibitors or ARBs will be challenging, but research in this area is abundant. Several new nonpeptide drugs have been developed that modify RAAS activity (Box 2); however, most of them are at preclinical or early clinical stages.

Inhibitors of the ACE-Ang II-AT1-aldosterone axis Angiotensin-receptor-neprilysin inhibitors

Natriuretic peptides have an important role in sodium and water homeostasis, inducing natriuresis, inhibiting
Box 1 | Effects mediated by key receptors of the RAAS

AT1 activation

- Sodium and water retention

- Vasoconstriction

- Inflammation

- Hypertension

- Aldosterone secretion

- Adrenocorticotropin release

- Prolactin release

- Vasopressin release

- Myocyte hypertrophy

- Fibrosis

- Endothelial dysfunction

- Inflammation

- Increased sympathetic nerve activity

- Thirst

AT2 activation

- Antiproliferative effects

- Apoptosis

- Vasodilation (masked by AT1)

- Antifibrotic effects

- Anti-inflammatory effects

- Neuroprotection and neuroregeneration

- Nitric oxide release

MAS1 activation

- Antifibrotic effects

- Anti-inflammatory effects

- Increased baroreflex sensitivity

- Antiproliferative effect on vascular smooth muscle cells

- Nitric oxide release

IRAP inhibition

- Memory and learning improvements

- Anti-inflammatory effects

- Antifibrotic effects

- Nitric oxide release

Abbreviations: AT1, type-1 angiotensin II receptor; AT2, type-2 angiotensin II receptor; IRAP, leucyl-cystinyl aminopeptidase (also known as insulin-regulated membrane aminopeptidase or insulin-responsive aminopeptidase); MAS1, proto-oncogene Mas; RAAS, renin-angiotensin-aldosterone system.

the hypothalamic-pituitary-adrenal system at all regulatory levels ${ }^{67,68}$ and promoting vasodilatation; they also possess antiproliferative properties. ${ }^{69,70}$ Neprilysin is a vasopeptidase that metabolizes natriuretic peptides and other peptides, such as bradykinin. Neprilysin inhibition increases the plasma levels of natriuretic peptides, but has modest effects in reducing blood pressure. ${ }^{71}$ However, combining neprilysin with a RAAS blocker is a powerful tool to increase natriuresis and vasodilation. The first therapeutic agent in this class was omapatrilat, a vasopeptidase that inhibits both neprilysin and ACE. The cardiovascular effects of omapatrilat were encouraging, but a high rate of angioedema stopped clinical trials of this drug. ${ }^{72}$ The next generation of drugs in this group combines a natriuretic peptide inhibitor with an ARB. LCZ696, the first agent of the angiotensinreceptor-neprilysin (ARN) inhibitor class, combines a neprilysin inhibitor moiety with a valsartan moiety. This dual therapy decreases blood pressure in animals and healthy humans with low rates of adverse effects. ${ }^{73}$ This type of drug intervention is promising in the fields of hypertension and heart failure. 


Box 2 | RAAS agonists and blockers
ACE inhibitors
- Benazepril, captopril, enalapril, lisinopril, fosinopril,
moexipril, perindopril, quinapril, ramipril, trandolapril
AT1 inhibitors
Classic ARBs
- Losartan, candesartan, irbesartan, olmesartan,
telmisartan, valsartan, eprosartan, fimasartan, azilsartan
ARN inhibitors
- LCZ696
Mineralocorticoid receptor antagonists
Steroidal
- Spironolactone, eplerenone, canrenone
Nonsteroidal
- Finerenone (BAY 94-8662), BR-4628, SM-368229
Aldosterone synthase inhibitors
- FAD 286 A, LCI699
Direct renin inhibitors
Classic
- Aliskiren
Novel
- ACT-077825, ACT-178882
AT2 agonists
- Compound 21
ACE2
- Human recombinant ACE2
MAS1 agonists
- AVE 0991, hydroxyl-propyl- - -cyclodextrin-Ang 1-7
- HFI-419
Abbreviations: ACE, angiotensin-converting enzyme;
ACE2, angiotensin-converting enzyme 2; Ang, angiotensin;
ARB, angiotensin receptor blocker; ARN, angiotensin receptor-
neprilysin; AT1, type-1 angiotensin II receptor; AT2, type-2
angiotensin II receptor; IRAP, leucyl-cystinyl aminopeptidase
(also known as insulin-regulated membrane aminopeptidase or
insulin-responsive aminopeptidase); MAS1, proto-oncogene Mas;
RAAS, renin-angiotensin-aldosterone system.
I

A randomized clinical trial (RCT) evaluated the effect of LCZ696 in the treatment of hypertension. ${ }^{71}$ Patients with mild to moderate hypertension $(n=1,212)$ received a single dose of 100,200 or $400 \mathrm{mg}$ of LCZ696, or 80,160 or $320 \mathrm{mg}$ of valsartan in a 12-week placebo-controlled trial. LCZ696 showed a good safety profile and was superior to placebo and valsartan in reducing office blood pressure and pulse pressure. More than $70 \%$ of patients had their blood pressure controlled with the $200 \mathrm{mg}$ dose of LCZ696, whereas this proportion was only $56 \%$ in the group receiving $160 \mathrm{mg}$ of valsartan. In approximately 50 patients per arm, ambulatory blood pressure monitoring was utilized. Mean diastolic $24 \mathrm{~h}$ blood pressure was not different between the groups receiving the two therapies, but mean systolic blood pressure was significantly reduced in the LCZ696 group because of larger nocturnal decreases in blood pressure than those observed in the valsartan or placebo groups. The greater blood pressure reduction seen in patients receiving LCZ696 when compared with patients in the other groups was not related to increased levels of the second messenger (cGMP) that mediates the actions of natriuretic peptides.
A second RCT in an Asian population also confirmed the efficacy of LCZ696 in treating hypertension. ${ }^{74}$ The PARAMETER study ${ }^{75}$ is an ongoing study in elderly patients with wide pulse pressure, which evaluates the vascular effects of ARN inhibition, including changes in arterial stiffness and central haemodynamics, and efficacy in reducing blood pressure. The trial will be finished in 2015.

The PARAMOUNT study ${ }^{76}$ evaluated the effects of $200 \mathrm{mg}$ of LCZ696 compared with $160 \mathrm{mg}$ of valsartan in almost 300 patients with heart failure and preserved ejection fraction. Even though the levels of $\mathrm{N}$-terminal proBNP (also known as B-type natriuretic peptide) were decreased and functional New York class of heart failure was improved at 36 weeks of follow-up, no differences in echocardiographic parameters were observed. No changes were observed in the levels of plasma inflammation markers. Surprisingly, proteinuria increased in the LCZ696 group, an increase that was not observed in patients treated with LCZ696 in the hypertension trials. This increase could be related to increased intraglomerular pressure related to increased levels of natriuretic peptides. ${ }^{77}$ The most important observations in this study were a $9 \mathrm{mmHg}$ decrease in systolic blood pressure and a $4 \mathrm{mmHg}$ decrease in diastolic blood pressure, which both persisted at 36 weeks of follow-up in the LCZ696 group, whereas the decreases in blood pressure seen in the valsartan group were $1.5 \mathrm{mmHg}$ and $0.3 \mathrm{mmHg}$, respectively, over the same period.

The PARADIGM-HF trial, in which therapy with $200 \mathrm{mg}$ of LCZ696 was compared with therapy with $10 \mathrm{mg}$ of enalapril for the treatment of heart failure (with decreased ejection fraction), was stopped according to prespecified rules because LCZ696 reduced the frequency of the primary outcome, a composite of death from cardiovascular causes or hospitalization for heart failure (HR 0.80, $P<0.001)^{78,79}$ Blood pressure was significantly lower in the LCZ969 group than in the enalapril group. The LCZ696 group had higher proportions of patients with hypertension and nonserious angioedema, but fewer patients with renal impairment and hyperkalaemia than the enalapril group. Urinary levels of cGMP in the LCZ969 group did not change during the trial. Some post-hoc analyses have noted that the benefits of LCZ969 in the treatment of heart failure do not correlate with the antihypertensive effects of the drug. ${ }^{80}$ ARN inhibition could be a breakthrough in the hypertension field. The observed reductions in systolic nocturnal blood pressure and in pulse pressure might be an indication of a substantial effect of LCZ969 on vascular function or on the central nervous system rather than an effect on natriuresis.

\section{Nonsteroidal mineralocorticoid receptor antagonists}

Nonsteroidal mineralocorticoid receptor antagonists (third-generation and fourth-generation compounds) have been developed with the aim of achieving cardiovascular benefits with fewer renal adverse effects than those of classic mineralocorticoid receptor antagonists (spironolactone, eplerenone and canrenone). 
Finerenone, previously called BAY $94-8862,{ }^{81}$ is a potent and promising mineralocorticoid receptor antagonist that conferred extensive cardiovascular and renal protection in rat models of hypertension and heart failure when compared with eplerenone. ${ }^{82}$ The ARTS trial assessed the efficacy and safety of this drug in patients with systolic heart failure and mild CKD. ${ }^{83}$ This phase II study consisted of two parts. Part A, which involved 65 patients, evaluated the safety and tolerability of 2.5, 5 or $10 \mathrm{mg}$ daily doses of finerenone versus placebo. Part B assessed the safety and efficacy of the drug in 392 patients randomized in a $1: 1: 1: 1: 1: 1$ design to receive finerenone orally at doses of $2.5,5$, or $10 \mathrm{mg}$ daily, or $5 \mathrm{mg}$ twice daily; placebo; or open-label oral spironolactone, which was given at an initial dose of $25 \mathrm{mg}$ daily and uptitrated to $50 \mathrm{mg}$ daily if serum potassium concentrations allowed. Finerenone decreased the levels of BNP and N-terminal proBNP. The drug decreased albuminuria by a similar degree to spironolactone and was associated with lower incidence of hyperkalaemia and acute renal failure. More data are needed to assess the clinical effects of finerenone. The ARTS HF trial, which has been completed but has not been published yet, assessed the efficacy and safety of this drug when compared with eplerenone in patients with diabetes mellitus but without CKD or chronic left-ventricular dysfunction. ${ }^{84}$

Another nonsteroidal drug, BR-4628, has high potency and selectivity for the mineralocorticoid receptor but also blocks the L-type calcium channel, which might help lowering blood pressure. After binding to the mineralocorticoid receptor, BR-4628 triggers degradation of this receptor. ${ }^{85}$ No clinical trials of BR-4628 have been reported. PF-3882845 is a potent and selective nonsteroidal inhibitor of the mineralocorticoid receptor that has been tested in healthy humans ${ }^{86,87}$ and patients with diabetic nephropathy, ${ }^{88}$ but was discontinued owing to adverse effects. SM-368229 is another agent of this class that has potent activity as an antagonist of the mineralocorticoid receptor and, in hypertensive rats, diminishes blood pressure and reduces the urinary levels of inflammatory markers. ${ }^{89}$ SM-368229 has not been tested in humans. ${ }^{90}$

\section{Aldosterone synthase inhibitors}

A different approach to avoid the deleterious effects of aldosterone is by inhibition of the expression of CYP11B2, the gene than encodes aldosterone synthase. Inhibition at this level of the RAAS would block the effects that are mediated through the mineralocorticoid receptor and also those that are independent of this receptor. An additional potential benefit of this approach is the prevention of a reactive increase in aldosterone levels, which occurs when the receptor is blocked. The potential downside of this strategy is that aldosterone synthase inhibitors might also suppress cortisol release by inhibiting cytochrome P450 11B1, mitochondrial (also known as steroid $11 \beta$-hydroxylase, encoded by the CYP11B1 gene), because the two enzymes have a high sequence homology.
FAD $286 \mathrm{~A}$ is an isomer of fadrozole, an aromatase inhibitor. This drug decreased plasma and urinary aldosterone concentrations and improved cardiac and renal target-organ damage in several animal models. ${ }^{91-93}$ FAD 286A has only a mild antihypertensive effect and, therefore, might be more clinically important in the treatment of heart failure than other approaches that have a more potent effect in lowering blood pressure.

LCI699 is an orally active and nonselective aldosterone synthase inhibitor that has been evaluated in human studies. In phase I studies, a $0.5 \mathrm{mg}$ daily dose of LCI699 was well tolerated and lowered plasma and urinary aldosterone levels without affecting cortisol secretion. ${ }^{94}$ At higher doses, however, cortisol secretion was diminished. Phase II studies demonstrated that plasma and urinary aldosterone levels are decreased with LCI699 therapy, but the RAAS is upregulated and slight hyponatraemia and increased serum potassium levels were reported.$^{95}$ In a large study, 524 patients were randomized to receive either LCI699 $0.25 \mathrm{mg}$ once daily $(n=92)$, $0.5 \mathrm{mg}$ once daily $(n=88), 0.5 \mathrm{mg}$ twice daily $(n=97)$ or $1.0 \mathrm{mg}$ once daily $(n=86)$; eplerenone $50 \mathrm{mg}$ twice daily $(n=84)$; or placebo $(n=77)$ for 8 weeks. ${ }^{96}$ LCI699 intake was associated with a modest reduction in blood pressure that was lower than that achieved with eplerenone. When aldosterone synthase is inhibited by LCI699, cortisol levels remain normal, but 11-deoxycorticosterone levels increase, which means that the adrenocorticotropichormone-cortisol axis is stimulated by the inhibition of expression of the CYP11B1 gene. ${ }^{97}$ The stimulation of this axis might be one of the reasons why the decrease in blood pressure is only modest even at high LCI699 doses.

Lack of aldosterone synthase selectivity, a short halflife and lack of adequate antihypertensive efficacy in primary aldosteronism and resistant hypertension hinder the usefulness of LCI699 in these areas. This agent, however, might be of value in the treatment of Cushing disease. ${ }^{98}$ Therefore, research is now set to develop second-generation aldosterone synthase inhibitors with better selectivity than LCI699, which would be associated with improved effects on reduction of blood pressure. $N$-(pyridin-3-yl) benzamides are potential agents for future development in this drug class. ${ }^{99,100}$

Post-transcriptional regulation of CYP11B1 and CYP11B2 expression by Dicer-dependent microRNAs (miRNAs) can affect secretion of aldosterone and cortisol in adrenocortical cells. ${ }^{101}$ Therefore, adrenal miRNAs might also be potential therapeutic targets.

\section{Direct renin inhibitors}

The rationale behind complete RAAS inhibition through blockade of the rate-limiting enzyme renin, and the expectation that this approach would more effectively prevent Ang II production than approaches targeted at other components of the RAAS ${ }^{102}$ is theoretically attractive. However, aliskiren is the only DRI available. As add-on therapy to ARBs, aliskiren did not improve renal or cardiovascular outcomes and was associated with more adverse effects than ARB therapy alone. ${ }^{58}$ Aliskiren has not been evaluated as initial or solo therapy, because 
other drugs have proven benefits when administered in this way. In the USA, the package insert of aliskiren contains a formal recommendation by the FDA that the drug should not be used as dual therapy in association with ARBs or ACE inhibitors in patients with diabetes mellitus and renal disease. In 2013, a new DRI, ACT-077825, was shown to have an adequate safety profile in humans when administered once daily. ${ }^{103}$ ACT-077825 inhibits plasma renin activity, while the levels of immunoreactive renin increase, a pattern typical of DRIs. ${ }^{102}$ However, the decrease in plasma renin activity is transient and is not dose-dependent at 7 days. ${ }^{103}$ The mechanism underlying these observations is not clear, because the plasma levels of ACT-077825 remain constant. ${ }^{103}$ The effects of this DRI on blood pressure are controversial, as only one study showed a decrease in blood pressure when ACT-077825 was used, and this effect was seen in the supine position, ${ }^{104}$ whereas other studies showed no differences between the effects of ACT-077825 and placebo on blood pressure in healthy individuals and patients with hypertension. ${ }^{103,105}$ More studies are necessary to clarify this inconsistency.

The same pharmaceutical company that developed ACT-077825 is developing another DRI, ACT-178882. In early phase I and phase II studies, ACT-178882 has shown a good safety profile. ${ }^{106}$ Not much information is yet available about the antihypertensive efficacy of ACT-178882.

\section{AT2 agonists}

For 20 years, different agonist and antagonist peptides were used to study the function of the AT2 receptor. The reported protective effects of this receptor were related to its antifibrotic and anti-inflammatory properties ${ }^{24}$ (Box 1). In 2004, a selective non-peptide AT2 agonist, Compound 21, was introduced for research. Most of the known effects of this agent have been described in different animal models. In rats with spontaneous hypertension, the drug reduces the amount of collagen in the extracellular matrix and in vascular tissue in a bloodpressure-independent manner. ${ }^{107}$ Compound 21 has a similar effect in animal models of myocardial infarction, reducing scar formation and improving cardiac function. ${ }^{108}$ Compound 21 reduced proteinuria in the kidney of rat models of hypertension and kidney disease, and also decreased the levels of inflammatory markers in these models. ${ }^{109,110}$ In the central nervous system, AT2 receptor activation has neuroprotective effects and induces neuroregeneration. Compound 21 reproduces these effects, but has to be administered centrally to bypass the blood-brain barrier. ${ }^{111}$ However, systemic administration of Compound 21 might still have effects on neuroregeneration. ${ }^{112}$ Compound 21 does not have a net effect on blood pressure, which is similar to the situation seen with other AT2 agonists. ${ }^{24}$ However, when the effects of the AT1 receptor on vasoconstriction are blocked, the vasodilator properties of Compound 21 are revealed, and blood pressure decreases. ${ }^{8.9}$ An AT2 antagonist can reverse this effect. A study published in 2014 demonstrated that the increased urinary sodium excretion that is induced by Compound 21 is mainly due to effects on proximal tubular cells of the kidney, namely internalization of sodium/ hydrogen exchanger 3 (NHE-3) and $\mathrm{Na}^{+} / \mathrm{K}^{+}$ATPase. ${ }^{113}$ Continuous Compound 21 infusion recruited more AT2 receptors to the proximal tubule brush border than an acute infusion. Acute Compound 21 infusion did not change blood pressure, but in a 7-day experiment in which concomitant intrarenal Ang II infusion was administered, Compound 21 blocked the blood pressure effects of Ang II, increasing natriuresis.

More studies are needed to clarify whether infusion time, time to achieve AT2 receptor upregulation, or administration route can influence blood pressure responses to Compound 21. Even though no clinical trials are published yet, available evidence suggests that Compound 21 could modulate fluid retention, hypertension and target-organ damage.

\section{ACE2-Ang 1-7-MAS1 axis} Human recombinant ACE2

The benefits of ACE2 in vascular disease might be brought about by two different mechanisms. First, by participating in Ang II degradation, ACE2 decreases the interaction of Ang II with AT1 receptors, and second, by increasing Ang 1-7 synthesis, ACE2 induces vasodilation. The main function of ACE2 has been proposed to be the counterbalancing of the effects of the ACEAng II-AT1-aldosterone axis. ${ }^{114}$ Decreases in ACE2 levels or increases in ACE and Ang II levels induced damage in animal models of diabetic nephropathy or hypertension. ${ }^{15,116}$ In animal models, the human recombinant form of ACE2 (rhACE2) reduced progression of diabetic nephropathy, decreased blood pressure (in models of hypertension), and decreased cardiac fibrosis. ${ }^{117,118}$ In 2013, the pharmacokinetic and pharmacodynamic characteristics of rhACE2 were described in healthy humans. ${ }^{119}$ Daily rhACE2 intake decreased the levels of Ang II in a dose-dependent manner over $24 \mathrm{~h}$, with a transient increase in Ang 1-7 levels, and more sustained increases in Ang 1-7 degradation products, such as Ang 1-5. The authors of this study suggest that rhACE2 is likely to reach the extravascular space, a hypothesis that is based on the large spread of rhACE2 distribution they observed. This enzyme has a good safety profile and did not induce immunogenicity, even when repeated doses were administered. Similarly to results reported in healthy animals, rhACE2 did not affect blood pressure or heart rate in humans. Thus, rhACE2 is a promising drug for treatment of patients with intolerance to other drugs that target RAAS components, and also perhaps in acute illnesses, such as myocardial infarction or decompensated heart failure, in which the balance between ACE and ACE2 is disturbed. This drug is attractive in the renal field, because rhACE2 might quickly reduce proteinuria, even in association with an ACE inhibitor. These two classes of drugs used together might have important protective effects mediated through substantial increases in Ang 1-7 levels. We look forward to more human clinical trials in this area. Aside from cardiovascular effects, some evidence exists that ACE2 might facilitate recovery from acute lung injury, as rhACE2 has been reported 
to ameliorate virus-mediated lung injury in mouse models. ${ }^{120}$ Thus, rhACE2 therapy in virus-mediated lung injury, especially influenza, in which Ang II levels are correlated with the degree of lung injury and with mortality, ${ }^{121}$ is potentially promising.

\section{MAS1 agonists}

Similarly to ACE2 administration, Ang 1-7 binding to the MAS1 receptor has shown beneficial effects in animal models; these effects are related to decreased fibrosis and inflammation and to blood pressure reduction by vasodilation and enhancement of baroreflex sensitivity. ${ }^{44}$ More than 10 years ago, the non-peptide compound AVE 0991 showed similar effects to those of Ang 1-7 in endothelial cells. ${ }^{122}$ Subsequent data demonstrated that AVE 0991 and Ang 1-7 compete for the same MAS1 receptor in the kidney. ${ }^{123}$ This non-peptide MAS1 receptor agonist has been studied in animals, in which it demonstrated a capacity to decrease blood pressure, alone or in combination with renin inhibitors. ${ }^{124}$ AVE 0991 was also demonstrated to attenuate acute and chronic renal injury in animal models. ${ }^{125,126}$ In 2014, AVE 0991 was shown to improve penile erection through nitric oxide release in rats. ${ }^{127}$ AVE 0991 has also been shown to ameliorate kidney inflammation, ${ }^{125}$ and, more interestingly, improve cell infiltration, cytokine release and histology in two rodent models of arthritis. ${ }^{128}$ Unfortunately, no clinical trials have yet been performed to test this promising therapeutic approach in humans.

The development of compounds that protect Ang 1-7 from enzymatic degradation has also been the focus of interest. The combination of Ang 1-7 with hydroxylpropyl- $\beta$-cyclodextrin protects Ang 1-7 from degradation and acts as a long-lasting release system, enabling uptake of Ang 1-7 in the colon. This drug combination has been tested in animal models of hypertension, in which it showed antihypertensive effects, ${ }^{129}$ and also had beneficial effects in models of diabetes mellitus and atherosclerotic inflammation. ${ }^{130,131}$ Phase I clinical trials are currently testing the safety profile of this combination therapy in humans (R. A. Santos, personal communication).

\section{IRAP inhibitors}

Many oligopeptides result from Ang II degradation by endopeptidases. The hexapeptide Ang 3-8, known as Ang IV, has been studied because it might have a role in protecting cognitive function..$^{29}$ IRAP, the Ang IV receptor, which is inhibited by Ang IV, was identified in $2001 .^{132}$ IRAP colocalizes with the glucose transporter GLUT-4 and was originally thought to contribute to basal intracellular retention of the transporter. ${ }^{133}$ IRAP is linked to vasopressin and oxytocin degradation. A group of nonpeptide inhibitors of IRAP has been developed. HFI-419 is an IRAP-selective pyridinyl compound that enhances memory in rats. ${ }^{134}$ An Australian group is working on the cardiovascular-protective effects of IRAP inhibition with HFI-419 in mice. Preliminary data demonstrate that HFI-419 prevents cardiac and endothelial damage induced by Ang II, independent of blood pressure. ${ }^{32}$ HFI-419 also has anti-inflammatory properties. ${ }^{32}$ The association of IRAP with GLUT-4 is intriguing, and whether IRAP inhibitors could be used to improve insulin sensitivity remains an interesting opportunity. More studies are necessary to explore therapeutic uses for IRAP inhibitors.

\section{Vaccines against the RAAS}

The high prevalence of hypertension in the world and the related cost of this burden have made the development of vaccines against the RAAS an attractive strategy, with the aim of achieving increased treatment compliance and public access to therapy, and a decrease in public-health costs. Many attempts were made to generate vaccines against renin, Ang I, Ang II and AT1.${ }^{135}$ However, most of these attempts have been harmful or have not had clinical efficacy. An RCT in which an anti-Ang II vaccine (CYT006-AngQb) was tested passed phase IIa without safety problems, and showed a promising $24 \mathrm{~h}$ reduction in blood pressure of $9.0 \mathrm{mmHg}$ (systolic) and $4.0 \mathrm{mmHg}$ (diastolic). ${ }^{136}$ However, subsequent studies could not confirm these results. Novel strategies are currently in development, such as the association of Ang II as an epitope to a hepatitis A virus-like particle to generate an improved immune response. ${ }^{137}$ Future studies are needed to explore the efficacy and safety of an immunological, chronic and irreversible blockade of the RAAS.

\section{Conclusions}

Although to improve upon the cardiovascular and renal protection offered by ACE inhibitors and ARBs will be difficult, novel drugs are being developed to shift the activity of the RAAS towards the protective cardiovascular arm (AT2 and MAS1 receptors). A role for RAAS blockade in other, non-cardiovascular pathologies (such as lung injury and cognitive disorders) is emerging. The combination of RAAS inhibition with other autacoid systems (endothelin blockade or natriuretic peptide stimulation) could improve the efficacy of traditional RAAS blockade and be the basis of specific tissue-targeted drug development.
1. Braun-Menendez, E., Fasciolo, J. C., Leloir, L. F. \& Munoz, J. M. The substance causing renal hypertension. J. Physiol. 98, 283-298 (1940).

2. Skeggs, L. T. Jr, Marsh, W. H., Kahn, J. R. \& Shumway, N. P. The existence of two forms of hypertensin. J. Exp. Med. 99, 275-282 (1954).

3. Nasjletti, A. \& Masson, G. M. Studies on angiotensinogen formation in a liver perfusion system. Circ. Res. 31 (Suppl. 2), 187 (1972).

4. Mulrow, P. J. \& Ganong, W. F. Stimulation of aldosterone secretion by angiotensin II.
A preliminary report. Yale J. Biol. Med. 33, 386-395 (1961).

5. Oparil, S., Tregear, G. W., Koerner, T., Barnes, B. A. \& Haber, E. Mechanism of pulmonary conversion of angiotensin I to angiotensin II in the dog. Circ. Res. 29, 682-690 (1971).

6. Hall, J. E. Historical perspective of the reninangiotensin system. Mol. Biotechnol. 24, 27-39 (2003).

7. Burns, K. D. \& Li, N. The role of angiotensin IIstimulated renal tubular transport in hypertension. Curr. Hypertens. Rep. 5, 165-171 (2003).

8. $\mathrm{Li}, \mathrm{H}$. et al. Renal proximal tubule angiotensin AT1A receptors regulate blood pressure. Am. J. Physiol. Regul. Integr. Comp. Physiol. 301 R1067-R1077 (2011).

9. Mamenko, M. et al. Chronic angiotensin II infusion drives extensive aldosteroneindependent epithelial $\mathrm{Na}^{+}$channel activation. Hypertension 62, 1111-1122 (2013). 
10. Beckerhoff, R. et al. Effect of angiotensin II and of an angiotensin II analogue (Sar1-Ile8-angiotensin II) on blood pressure, plasma aldosterone and plasma renin activity in the dog. Clin. Sci. Mol. Med. Suppl. 2, 41s-44s (1975).

11. Kakiki, M., Morohashi, K., Nomura, M., Omura, T. \& Horie, T. Expression of aldosterone synthase cytochrome P450 (P450aldo) mRNA in rat adrenal glomerulosa cells by angiotensin II type 1 receptor. Endocr. Res. 23, 277-295 (1997).

12. Fuller, P. J. \& Young, M. J. Mechanisms of mineralocorticoid action. Hypertension 46 1227-1235 (2005).

13. Pfeffer, M. A. et al. Effect of captopril on mortality and morbidity in patients with left ventricular dysfunction after myocardial infarction. Results of the survival and ventricular enlargement trial. The SAVE Investigators. N. Engl. J. Med. 327, 669-677 (1992).

14. Lewis, E. J. et al. Renoprotective effect of the angiotensin-receptor antagonist irbesartan in patients with nephropathy due to type 2 diabetes. N. Engl. J. Med. 345, 851-860 (2001).

15. Rigatto, C. et al. Congestive heart failure in renal transplant recipients: risk factors, outcomes, and relationship with ischemic heart disease. J. Am. Soc. Nephrol. 13, 1084-1090 (2002).

16. Yusuf, S. et al. Effects of an angiotensinconverting-enzyme inhibitor, ramipril, on cardiovascular events in high-risk patients. The Heart Outcomes Prevention Evaluation Study Investigators. N. Engl. J. Med. 342, 145-153 (2000).

17. Brenner, B. M. et al. Effects of losartan on renal and cardiovascular outcomes in patients with type 2 diabetes and nephropathy. N. Engl. J. Med. 345, 861-869 (2001).

18. Danser, A. H. et al. Determinants of interindividual variation of renin and prorenin concentrations: evidence for a sexual dimorphism of (pro)renin levels in humans. J. Hypertens. 16, 853-862 (1998).

19. Nguyen, G. Renin/prorenin receptors. Kidney Int 69, 1503-1506 (2006)

20. Schefe, J. H. et al. A novel signal transduction cascade involving direct physical interaction of the renin/prorenin receptor with the transcription factor promyelocytic zinc finger protein. Circ. Res. 99, 1355-1366 (2006).

21. Krop, M., Lu, X., Danser, A. H. \& Meima, M. E. The (pro)renin receptor. A decade of research: what have we learned? Pflugers Arch. 465 87-97 (2013).

22. Shricker, K., Holmer, S., Kramer, B. K., Riegger, G. A. \& Kurtz, A. The role of angiotensin II in the feedback control of renin gene expression. Pflugers Arch. 434, 166-172 (1997).

23. Dandona, P., Dhindsa, S., Ghanim, H. \& Chaudhuri, A. Angiotensin II and inflammation: the effect of angiotensin-converting enzyme inhibition and angiotensin II receptor blockade. J. Hum. Hypertens. 21, 20-27 (2007).

24. Steckelings, U. M., Paulis, L., Namsolleck, P. \& Unger, T. AT2 receptor agonists: hypertension and beyond. Curr. Opin. Nephrol. Hypertens. 21, 142-146 (2012).

25. Tipnis, S. R. et al. A human homolog of angiotensin-converting enzyme. Cloning and functional expression as a captopril-insensitive carboxypeptidase. J. Biol. Chem. 275, 33238-33243 (2000).

26. Chappell, M. C., Marshall, A. C., Alzayadneh, E. M., Shaltout, H. A. \& Diz, D. I. Update on the angiotensin converting enzyme 2-Angiotensin (1-7)-MAS receptor axis: fetal programing, sex differences, and intracellular pathways. Front. Endocrinol. (Lausanne) 4, 201 (2014).

27. Santos, R. A., Ferreira, A. J., Verano-Braga, T. $\&$ Bader, M. Angiotensin-converting enzyme 2, angiotensin-(1-7) and Mas: new players of the renin-angiotensin system. J. Endocrinol. 216, R1-R17 (2013).

28. Sanchez-Agesta Ortega, R. et al. Circulating aminopeptidase activities in men and women with essential hypertension. Curr. Med. Chem. 20, 4935-4945 (2013).

29. Andersson, H. \& Hallberg, M. Discovery of inhibitors of insulin-regulated aminopeptidase as cognitive enhancers. Int. J. Hypertens. 2012 789671 (2012).

30. Vinh, A., Widdop, R. E., Chai, S. Y. \& Gaspari, T. A. Angiotensin IV-evoked vasoprotection is conserved in advanced atheroma. Atherosclerosis 200, 37-44 (2008).

31. Vinh, A., Widdop, R. E., Drummond, G. R. \& Gaspari, T. A. Chronic angiotensin IV treatment reverses endothelial dysfunction in ApoE-deficient mice. Cardiovasc. Res. 77, 178-187 (2008).

32. Lee, H. W. R., Chai, S., Pong, W., Welungoda, I. \& Gaspari, T. AT4 receptor/insulin regulated aminopeptidase inhibition protects against angiotensin II-induced cardic fibrosis and vascular dysfunction [abstract 33.39]. J. Hypertens. 32 (Suppl. 1), e551 (2014).

33. Gonçalves, P. B., Ferreira, R., Gasperin, B. \& Oliveira, J. F. Role of angiotensin in ovarian follicular development and ovulation in mammals: a review of recent advances. Reproduction 143, 11-20 (2012).

34. Zhang, Z. et al. Improved glucose-stimulated insulin secretion by selective intraislet inhibition of angiotensin II type 1 receptor expression in isolated islets of $d b / d b$ mice. Int. J. Endocrinol. 2013, 319586 (2013)

35. Paul, M., Poyan Mehr, A. \& Kreutz, R. Physiology of local renin-angiotensin systems. Physiol. Rev. 86, 747-803 (2006).

36. Jurewicz, M. et al. Human T and natural killer cells possess a functional renin-angiotensin system: further mechanisms of angiotensin Il-induced inflammation. J. Am. Soc. Nephrol. 18, 1093-1102 (2007)

37. Chen, Y. H. et al. Prognostic impact of reninangiotensin system blockade in esophageal squamous cell carcinoma. J. Renin Angiotensin Aldosterone Syst. http://dx.doi.org/ 10.1177/1470320314535275.

38. Nakai, Y. et al. Inhibition of renin-angiotensin system affects prognosis of advanced pancreatic cancer receiving gemcitabine. Br. J. Cancer 103, 1644-1648 (2010).

39. Iwanami, J., Mogi, M., Iwai, M. \& Horiuchi, M. Inhibition of the renin-angiotensin system and target organ protection. Hypertens. Res. 32, 229-237 (2009).

40. [No authors listed] Effect of enalapril on survival in patients with reduced left ventricular ejection fractions and congestive heart failure. The SOLVD Investigators. N. Engl. J. Med. 325, 293-302 (1991).

41. Dzau, V. J. et al. Sustained effectiveness of converting-enzyme inhibition in patients with severe congestive heart failure. N. Engl. J. Med. 302, 1373-1379 (1980).

42. Gonzalez, G. E. et al. N-acetyl-seryl-aspartyl-lysylproline reduces cardiac collagen cross-linking and inflammation in angiotensin II-induced hypertensive rats. Clin. Sci. (Lond.) 126, 85-94 (2014).

43. Luque, M. et al. Effects of captopril related to increased levels of prostacyclin and angiotensin-(1-7) in essential hypertension. J. Hypertens. 14, 799-805 (1996).
44. Santos, R. A. Angiotensin-(1-7). Hypertension 63, 1138-1147 (2014).

45. Klein, W. S., Merz, C. N. \& Jenkins, M. R. Guidelines for managing high blood pressure. JAMA 312, 294-295 (2014).

46. Krause, T., Lovibond, K., Caulfield, M., McCormack, T. \& Williams, B. Management of hypertension: summary of NICE guidance. BMJ 343, d4891 (2011).

47. Go, A. S. et al. An effective approach to high blood pressure control: a science advisory from the American Heart Association, the American College of Cardiology, and the Centers for Disease Control and Prevention. Hypertension 63, 878-885 (2014).

48. Fagard, R. H., Celis, H., Thijs, L. \& Wouters, S. Regression of left ventricular mass by antihypertensive treatment: a meta-analysis of randomized comparative studies. Hypertension 54, 1084-1091 (2009).

49. van Vark, L. C. et al. Angiotensin-converting enzyme inhibitors reduce mortality in hypertension: a meta-aalysis of radomized clinical trials of renin-angiotensin-aldosterone system inhibitors involving 158,998 patients. Eur. Heart J. 33, 2088-2097 (2012).

50. Michel, M. C., Foster, C., Brunner, H. R. \& Liu, L. A systematic comparison of the properties of clinically used angiotensin II type 1 receptor antagonists. Pharmacol. Rev. 65, 809-848 (2013).

51. Toh, S. et al. Comparative risk for angioedema associaed with the se of drugs that target the renin-angiotensin-aldosterone system. Arch. Intern. Med. 172, 1582-1589 (2012).

52. Dahlof, B. et al. Cardiovascular morbidity and mortality in the Losartan Intervention For Endpoint reduction in hypertension study (LIFE): a randomised trial against atenolol. Lancet 359 995-1003 (2002).

53. Yusuf, S. et al. Effects of the angiotensinreceptor blocker telmisartan on cardiovascular events in high-risk patients intolerant to angiotensin-converting enzyme inhibitors: a randomised controlled trial. Lancet $\mathbf{3 7 2}$, 1174-1183 (2008).

54. Yusuf, S. et al. Telmisartan, ramipril, or both in patients at high risk for vascular events. N. Engl. J. Med. 358, 1547-1559 (2008).

55. Pitt, B. et al. Effect of losartan compared with captopril on mortality in patients with symptomatic heart failure: randomised trial -the Losartan Heart Failure Survival Study ELITE II. Lancet 355, 1582-1587 (2000).

56. McMurray, J. J. et al. Effects of candesartan in patients with chronic heart failure and reduced left-ventricular systolic function taking angiotensin-converting-enzyme inhibitors: the CHARM-Added trial. Lancet 362, 767-771 (2003).

57. Barnett, A. H. et al. Angiotensin-receptor blockade versus converting-enzyme inhibition in type 2 diabetes and nephropathy. N. Engl. J. Med. 351, 1952-1961 (2004).

58. Parving, H. H. et al. Cardiorenal end points in a trial of aliskiren for type 2 diabetes. N. Engl. J. Med. 367, 2204-2213 (2012).

59. Fried, L. F. et al. Combined angiotensin inhibition for the treatment of diabetic nephropathy. N. Engl. J. Med. 369, 1892-1903 (2013)

60. Zannad, F. et al. Eplerenone in patients with systolic heart failure and mild symptoms. N. Engl. J. Med. 364, 11-21 (2011).

61. Pitt, B. et al. Eplerenone, a selective aldosterone blocker, in patients with left ventricular dysfunction after myocardial infarction. N. Engl. J. Med. 348, 1309-1321 (2003). 
62. Armanini, D., Sabbadin, C., Dona, G., Clari, G. \& Bordin, L. Aldosterone receptor blockers spironolactone and canrenone: two multivalent drugs. Expert Opin. Pharmacother. 15, 909-912 (2014).

63. Runyon, B. A. Introduction to the revised American Association for the Study of Liver Diseases Practice Guideline management of adult patients with ascites due to cirrhosis 2012. Hepatology 57, 1651-1653 (2013).

64. Pitt, B. et al. The effect of spironolactone on morbidity and mortality in patients with severe heart failure. Randomized Aldactone Evaluation Study Investigators. N. Engl. J. Med. 341, 709-717 (1999).

65. Oxlund, C. S. et al. Low dose spironolactone reduces blood pressure in patients with resistant hypertension and type 2 diabetes mellitus: a double blind randomized clinical trial. J. Hypertens. 31, 2094-2102 (2013).

66. de Souza, F., Muxfeldt, E., Fiszman, R. \& Salles, G. Efficacy of spironolactone therapy in patients with true resistant hypertension. Hypertension 55, 147-152 (2010).

67. Porzionato, A., Macchi, V., Rucinski, M. Malendowicz, L. K. \& De Caro, R. Natriuretic peptides in the regulation of the hypothalamicpituitary-adrenal axis. Int. Rev. Cell. Mol. Biol. 280, 1-39 (2010).

68. Wiedemann, K., Jahn, H. \& Kellner, M. Effects of natriuretic peptides upon hypothalamopituitary-adrenocortical system activity and anxiety behaviour. Exp. Clin. Endocrinol. Diabetes 108, 5-13 (2000).

69. Gardner, D. G., Chen, S., Glenn, D. J. \& Grigsby, C. L. Molecular biology of the natriuretic peptide system: implications for physiology and hypertension. Hypertension 49, 419-426 (2007)

70. Pandey, K. N. Biology of natriuretic peptides and their receptors. Peptides 26, 901-932 (2005).

71. Ruilope, L. M. et al. Blood-pressure reduction with LCZ696, a novel dual-acting inhibitor of the angiotensin II receptor and neprilysin: a randomised, double-blind, placebo-controlled, active comparator study. Lancet $\mathbf{3 7 5}$, 1255-1266 (2010).

72. Kostis, J. B. et al. Omapatrilat and enalapril in patients with hypertension: the Omapatrilat Cardiovascular Treatment vs. Enalapril (OCTAVE) trial. Am. J. Hypertens. 17, 103-111 (2004).

73. Gu, J. et al. Pharmacokinetics and pharmacodynamics of LCZ696, a novel dualacting angiotensin receptor-neprilysin inhibitor (ARNi). J. Clin. Pharmacol. 50, 401-414 (2010).

74. Kario, K. et al. Efficacy and safety of LCZ696, a first-in-class angiotensin receptor neprilysin inhibitor, in Asian patients with hypertension: a randomized, double-blind, placebo-controlled study. Hypertension 63, 698-705 (2014).

75. Williams, B. et al. Rationale and study design of the Prospective comparison of Angiotensin Receptor neprilysin inhibitor with Angiotensin receptor blocker MEasuring arterial sTiffness in the eldERly (PARAMETER) study. BMJ Open 4 , e004254 (2014).

76. Solomon, S. D. et al. The angiotensin recepto neprilysin inhibitor LCZ696 in heart failure with preserved ejection fraction: a phase 2 doubleblind randomised controlled trial. Lancet 380 1387-1395 (2012)

77. Marin-Grez, M., Fleming, J. T. \& Steinhausen, M. Atrial natriuretic peptide causes pre-glomerular vasodilatation and post-glomerular vasoconstriction in rat kidney. Nature $\mathbf{3 2 4}$, 473-476 (1986).

78. McMurray, J. J. et al. Angiotensin-neprilysin inhibition versus enalapril in heart failure. N. Engl. J. Med. 371, 993-1004 (2014).
79. McMurray, J. J. et al. Baseline characteristics and treatment of patients in Prospective comparison of ARNI with ACEI to Determine Impact on Global Mortality and morbidity in Heart Failure trial (PARADIGM-HF). Eur. J. Heart Fail. 16, 817-825 (2014).

80. Jhund, P. S. et al. Independence of the blood pressure lowering effect and efficacy of the angiotensin receptor neprilysin inhibitor, LCZ696, in patients with heart failure with preserved ejection fraction: an analysis of the PARAMOUNT trial. Eur. J. Heart Fail. 16, 671-677 (2014).

81. Barfacker, L. et al. Discovery of BAY 94-8862: a nonsteroidal antagonist of the mineralocorticoid receptor for the treatment of cardiorenal diseases. ChemMedChem 7 , 1385-1403 (2012)

82. Kolkhof, P. et al. Finerenone, a novel selective nonsteroidal mineralocorticoid receptor antagonist protects from rat cardiorenal injury. J. Cardiovasc. Pharmacol. 64, 69-78 (2014).

83. Pitt, B. et al. Safety and tolerability of the novel non-steroidal mineralocorticoid receptor antagonist BAY 94-8862 in patients with chronic heart failure and mild or moderate chronic kidney disease: a randomized, double-blind trial. Eur. Heart J. 34, 2453-2463 (2013).

84. US National Library of Medicine. Clinicaltrials.gov [online], https://www.clinicaltrials.gov/ct2/ show?term=ARTS+HF\&rank=1 (2014).

85. Fagart, J. et al. A new mode of mineralocorticoid receptor antagonism by a potent and selective nonsteroidal molecule. J. Biol. Chem. 285 29932-29940 (2010)

86. US National Library of Medicine. Clinicaltrials.gov [online], https://www.clinicaltrials.gov/ct2/ show/NCT00971802?term=NCT00971802\& rank=1 (2010)

87. US National Library of Medicine. Clinicaltrials.gov [online], https://www.clinicaltrials.gov/ct2/ show/NCT00856258?term $=$ NCT008562588 rank=1 (2009).

88. US National Library of Medicine. Clinicaltrials.gov [online], https://www.clinicaltrials.gov/ct2/ show/NCT01488877?term=NCT01488877\& rank=1 (2013).

89. Nariai, T. et al. SM-368229, a novel selective and potent non-steroidal mineralocorticoid receptor antagonist with strong urinary $\mathrm{Na}^{+}$ excretion activity. J. Pharmacol. Sci. 115, 346-353 (2011).

90. Nariai, T. et al. Antihypertensive and cardiorenal protective effects of SM-368229, a novel mineralocorticoid receptor antagonist, in aldosterone/salt-treated rats. Pharmacology 89 , 44-52 (2012).

91. Menard, J., Gonzalez, M. F., Guyene, T. T. \& Bissery, A. Investigation of aldosterone-synthase inhibition in rats. J. Hypertens. 24, 1147-1155 (2006).

92. Lea, W. B. et al. Aldosterone antagonism or synthase inhibition reduces end-organ damage induced by treatment with angiotensin and high salt. Kidney Int. 75, 936-944 (2009).

93. Mulder, P. et al. Aldosterone synthase inhibition improves cardiovascular function and structure in rats with heart failure: a comparison with spironolactone. Eur. Heart J. 29, 2171-2179 (2008).

94. Amar, L. et al. Aldosterone synthase inhibition with LCI699: a proof-of-concept study in patients with primary aldosteronism. Hypertension $\mathbf{5 6}$ 831-838 (2010).

95. Andersen, K. et al. The effects of aldosterone synthase inhibition on aldosterone and cortisol in patients with hypertension: a phase II, randomized, double-blind, placebo-controlled, multicenter study. J. Clin. Hypertens. (Greenwich) 14, 580-587 (2012)

96. Calhoun, D. A. et al. Effects of a novel aldosterone synthase inhibitor for treatment of primary hypertension: results of a randomized, double-blind, placebo- and active-controlled phase 2 trial. Circulation 124, 1945-1955 (2011)

97. Amar, L. et al. Aldosterone synthase inhibition with LCl699: a proof-of-concept study in patients with primary aldosteronism. Hypertension 56, 831-838 (2010).

98. Bertagna, X. et al. LCI699, a potent $11 \beta$-hydroxylase inhibitor, normalizes urinary cortisol in patients with Cushing's disease: results from a multicenter, proof-of-concept study. J. Clin. Endocrinol. Metab. 99, 1375-1383 (2014).

99. Zimmer, C. et al. N-(Pyridin-3-yl)benzamides as selective inhibitors of human aldosterone synthase (CYP11B2). Bioorg. Med. Chem. Lett. 21, 186-190 (2011).

100. Azizi, M., Amar, L. \& Menard, J. Aldosterone synthase inhibition in humans. Nephrol. Dial. Transplant. 28, 36-43 (2013).

101. Robertson, S. et al. MicroRNA-24 is a novel regulator of aldosterone and cortisol production in the human adrenal cortex. Hypertension 62, 572-578 (2013)

102. Staessen, J. A., Li, Y. \& Richart, T. Oral renin inhibitors. Lancet 368, 1449-1456 (2006).

103. Nicolas, L. B., Gutierrez, M., Binkert, C. \& Dingemanse, J. Pharmacokinetics, pharmacodynamics, and tolerability of ACT-077825, a new direct renin inhibitor after multiple-ascending doses in healthy subjects. J. Cardiovasc. Pharmacol. 61, 42-50 (2013).

104. Nicolas, L. B., Gutierrez, M. M., Binkert, C. \& Dingemanse, J. Entry-into-humans study with a new direct renin inhibitor. Eur. J. Clin. Pharmacol. 68, 1257-1266 (2012)

105. Jones-Burton, C. et al. Effects of the renin inhibitor MK-8141 (ACT-077825) in patients with hypertension. J. Am. Soc. Hypertens. 4, 219-226 (2010).

106. Dingemanse, J. \& Nicolas, L. Age and sex effects on the single- and multiple-dose safety and pharmacokinetics of the new renin inhibitor ACT-178882. Cardiovasc. Ther. 31, 307-313 (2013)

107. Rehman, A. et al. Angiotensin type 2 receptor agonist compound 21 reduces vascular injury and myocardial fibrosis in stroke-prone spontaneously hypertensive rats. Hypertension 59, 291-299 (2012)

108. Kaschina, E. et al. Angiotensin II type 2 receptor stimulation: a novel option of therapeutic interference with the renin-angiotensin system in myocardial infarction? Circulation 118, 2523-2532 (2008)

109. Gelosa, P. et al. Stimulation of AT2 receptor exerts beneficial effects in stroke-prone rats: focus on renal damage. J. Hypertens. 27 , 2444-2451 (2009).

110. Matavelli, L. C., Huang, J. \& Siragy, H. M. Angiotensin AT(2) receptor stimulation inhibits early renal inflammation in renovascular hypertension. Hypertension 57, 308-313 (2011)

111. McCarthy, C. A. et al. Direct angiotensin AT2 receptor stimulation using a novel AT2 receptor agonist, compound 21, evokes neuroprotection in conscious hypertensive rats. PLOS ONE 9, e95762 (2014)

112. Namsolleck, P. et al. AT2-receptor stimulation enhances axonal plasticity after spinal cord injury by upregulating BDNF expression. Neurobiol. Dis. 51, 177-191 (2013). 
113. Kemp, B. A. et al. AT2 receptor activation induces natriuresis and lowers blood pressure. Circ. Res. 115, 388-399 (2014).

114. Clarke, N. E. \& Turner, A. J. Angiotensinconverting enzyme 2: the first decade. Int. J. Hypertens. 2012, 307315 (2012).

115. Ye, M. et al. Glomerular localization and expression of angiotensin-converting enzyme 2 and angiotensin-converting enzyme: implications for albuminuria in diabetes. J. Am. Soc. Nephrol. 17, 3067-3075 (2006).

116. Rentzsch, B. et al. Transgenic angiotensinconverting enzyme 2 overexpression in vessels of SHRSP rats reduces blood pressure and improves endothelial function. Hypertension $\mathbf{5 2}$, 967-973 (2008).

117. Oudit, G. Y. et al. Human recombinant ACE2 reduces the progression of diabetic nephropathy. Diabetes 59, 529-538 (2010).

118. Zhong, J. et al. Angiotensin-converting enzyme 2 suppresses pathological hypertrophy, myocardial fibrosis, and cardiac dysfunction. Circulation 122, 717-728 (2010).

119. Haschke, M. et al. Pharmacokinetics and pharmacodynamics of recombinant human angiotensin-converting enzyme 2 in healthy human subjects. Clin. Pharmacokinet. 52, 783-792 (2013).

120. Zou, Z. et al. Angiotensin-converting enzyme 2 protects from lethal avian influenza A H5N1 infections. Nat. Commun. 5, 3594 (2014).

121. Huang, F. et al. Angiotensin II plasma levels are linked to disease severity and predict fatal outcomes in H7N9-infected patients. Nat. Commun. 5, 3595 (2014).

122. Wiemer, G., Dobrucki, L. W., Louka, F. R., Malinski, T. \& Heitsch, H. AVE 0991, a nonpeptide mimic of the effects of angiotensin-(1-7) on the endothelium. Hypertension 40, 847-852 (2002).

123. Pinheiro, S. V. et al. Nonpeptide AVE 0991 is an angiotensin-(1-7) receptor Mas agonist in the mouse kidney. Hypertension 44, 490-496 (2004).

124. Singh, Y., Singh, K. \& Sharma, P. L. Effect of combination of renin inhibitor and Masreceptor agonist in DOCA-salt-induced hypertension in rats. Mol. Cell. Biochem. 373, 189-194 (2013).

125. Barroso, L. C. et al. Renoprotective effects of AVE0991, a nonpeptide Mas receptor agonist, in experimental acute renal injury. Int. J. Hypertens. 2012, 808726 (2012).

126. Silveira, K. D. et al. Beneficial effects of the activation of the angiotensin-(1-7) MAS receptor in a murine model of adriamycininduced nepopathy. PLoS ONE 8, e66082 (2013).

127. da Costa Gonçalves, A. C., Fraga-Silva, R. A., Leite, R. \& Santos, R. A. AVE 0991, a non-peptide Mas-receptor agonist, facilitates penile erection. Exp. Physiol. 98, 850-855 (2013).

128. da Silveira, K. D. et al. Anti-inflammatory effects of the activation of the angiotensin-(1-7) receptor, MAS, in experimental models of arthritis. J. Immunol. 185, 5569-5576 (2010).

129. Bertagnolli, M. et al. An orally active angiotensin-(1-7) inclusion compound and exercise training produce similar cardiovascular effects in spontaneously hypertensive rats. Peptides 51, 65-73 (2014).

130. Fraga-Silva, R. A. et al. Treatment with angiotensin-(1-7) reduces inflammation in carotid atherosclerotic plaques. Thromb. Haemost. 111, 736-747 (2014).
131. Santos, S. H. et al. Oral administration of angiotensin-(1-7) ameliorates type 2 diabetes in rats. J. Mol. Med. (Berl.) 92, 255-265 (2014).

132. Albiston, A. L. et al. Evidence that the angiotensin IV (AT(4)) receptor is the enzyme insulin-regulated aminopeptidase. J. Biol. Chem. 276, 48623-48626 (2001).

133. Jordens, I., Molle, D., Xiong, W., Keller, S. R. \& McGraw, T. E. Insulin-regulated aminopeptidase is a key regulator of GLUT4 trafficking by controlling the sorting of GLUT4 from endosomes to specialized insulin-regulated vesicles. Mol. Biol. Cell 21, 2034-2044 (2010).

134. Albiston, A. L. et al. Identification and characterization of a new cognitive enhancer based on inhibition of insulin-regulated aminopeptidase. FASEB J. 22, 4209-4217 (2008).

135. Pandey, R., Quan, W. Y., Hong, F. \& Jie, S. L. Vaccine for hypertension: modulating the renin-angiotensin system. Int. J. Cardiol. 134, 160-168 (2009).

136. Tissot, A. C. et al. Effect of immunisation against angiotensin II with CYT006-AngQb on ambulatory blood pressure: a double-blind, randomised, placebo-controlled phase lla study. Lancet 371, 821-827 (2008).

137. Ou, X. et al. Construction, expression and immunogenicity of a novel anti-hypertension angiotensin II vaccine based on hepatitis A virus-like particle. Hum. Vaccin. Immunother. 9 , 1191-1199 (2013).

\section{Author contributions}

C.A.R. researched data for the article. C.A.R. and M.R.W. wrote the article. All authors contributed substantially to discussion of content and reviewing and/or editing the manuscript before submission. 